\title{
Light Source for Producing Self-Reversed Spectral Lines
}

\author{
Jack Sugar
}

(March 26, 1962)

\begin{abstract}
A new light source has been developed which produces numerous self-reversed lines in both the first and second spectra of rare-earth elements. It consists of a pulsed are discharge with a peak current of 75 amperes and an on-time of one millisecond per cycle. Resonance lines are nearly completely absorbed, and can be distinguished by this character. From spectrograms obtained with this light source, the ground states of Tb I and U II were determined, and those reported for $\mathrm{Yb}$ I, Yb II, Tm I, Tm II, and U I were confirmed.
\end{abstract}

\section{Introduction}

In atomic emission spectra, the presence of selfreversed spectral lines provides valuable information for the analysis of spectral structure. Because such lines generally arise from transitions to the ground state or to low-lying states of the atom, they may be used to deduce this important part of the energy level structure. In searching for regularities in a line-list containing tens of thousands of entries, as do those of most rare-earth elements, it is a great simplification to recognize a small group of lines which have energy levels in common.

In the past, at least three different light sources have been employed for observing the lines that are most readily absorbed or reversed; they are (1) the high-temperature furnace, (2) the underwater spark, and (3) the d-c are.

(1) A. S. King developed the technique of obtaining atomic absorption lines by photographing a continuous spectrum which has passed through an electrically heated carbon tube containing metal vapor. An early example of his work is the description of the absorption spectrum of chromium [1]. ${ }^{1}$ However, as a result of the relatively low temperature limitation on the a bsorbing vapor, only very low-lying levels of the neutral atom, and in general no ionic levels, are sufficiently populated to produce absorption lines. Furthermore, metals with very high boiling points will not yield to this technique.

(2) Spectral phenomena observed when highvoltage condensed sparks are passed between metallic electrodes submerged in water have been investigated for more than half a century. It was found that the dissociation of water produces a continuum in which a small fraction of the spectral lines characteristic of the metal vapor are absorbed. An example of the application of this technique to the determination of atomic energy levels is the study of the underwater absorption spectra of the palladium and platinum triads by W. F. Meggers and O. Laporte [2]. The main difficulties with this technique arise from

${ }_{1}^{1}$ Figures in brackets indicate the literature references at the end of this paper. the presence in some spectral regions of absorption bands due to water molecules, the superposition of metallic spark lines in emission, and the total absence of absorption for certain metals, presumably because of instant oxidation.

(3) A d-c are discharge with a current of several amperes nearly always produces self-reversal of the very strong lines in the spectrum of the electrode material by absorption in the sheath of cool vapor surrounding the discharge. The degree of selfreversal increases exponentially with the product of the intensity of the radiation and the number of absorbing atoms through which it passes [3].

The spectra of rare-earth elements are characteristically lacking in lines of outstanding high intensity. Therefore, they rarely contain selfreversed lines in the low-current arc. It has been found that by raising the current in the arc, one usually enhances self-reversal because of the increased rate of vapor production. This increases the density of absorbing atoms surrounding the discharge. A substantial rise in current, however, will generally melt the electrodes unless adequate cooling is provided. A convenient method for obtaining a cool, high-current are is described in this paper.

\section{Apparatus}

A square current pulse of short duration and high ampereage may be obtained by discharging a delay line through a matching load impedance. This may be employed to produce a pulsed high-current arc by placing the electrode gap in series with the load. If a suitable pulse width and repetition rate is selected, the average current can be kept low enough so that no cooling is required to prevent the electrodes from melting.

A diagram of the electrical circuit used in this experiment is given in figure 1. A delay line consisting of five stages with a capacitance of $20 \mu \mathrm{f}$ and an inductance of $500 \mu \mathrm{h}$ per stage was charged to $400 \mathrm{v}$. The rotating shorting bar in series with the load was employed to close the output circuit, 


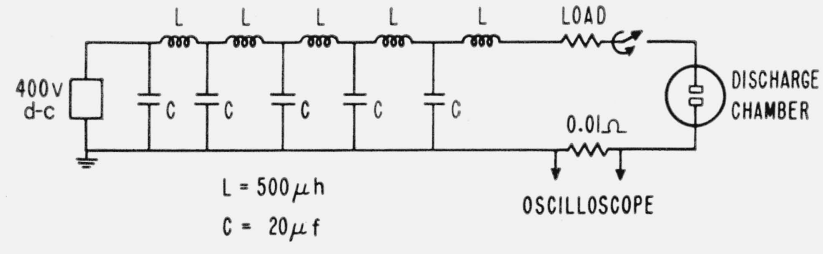

Figure 1. Electrical circuit for obtaining pulsed arc discharge.

discharging the delay line through the arc chamber. The proper load resistor was determined by observing the voltage waveform across the $0.01 \mathrm{ohm}$ resistor with an oscilloscope. Too little resistance results in an oscillating discharge, while too much causes a distorted pulse shape. With the above circuit values, the load was about 2 ohms, and a square pulse of 75 amp peak and 1 msec duration was obtained.

A repetitive rate of 10 per second gave an average current of only $0.75 \mathrm{amp}$ while providing a strong exposure in about 5 to $10 \mathrm{~min}$ in the wavelength range 2400 to $7000 \mathrm{~A}$. The spectrograms were made with a Wadsworth mounting of a $4 \times 2$ in. concave grating having a $21 \mathrm{ft}$ radius and 30,000 grooves per in.

The discharge chamber contained helium at a pressure of $1 \mathrm{~atm}$. The operation of this arc in an inert atmosphere prevents the oxidation of metal vapor so that an absorbing layer can be maintained. Furthermore, the presence of highly energetic helium metastable atoms favors the production of metallic ions so that strong absorption is exhibited by these ions as well as by the neutral atoms.

A gap of about $2 \mathrm{~mm}$ was maintained between the electrodes.

\section{Observations}

The spectra of ytterbium, thulium, uranium, and terbium were obtained with the pulsed arc. Portions of each of these are shown in figure 2 , where the self-reversed lines are indicated by a dot and the resonance lines of the neutral and singly-ionized atom are further designated by $R_{I}$ and $R_{I I}$, respectively. Numerous self-reversed lines appeared among the first spectrum lines of each of these elements and among the second spectrum lines of all but uranium, which contained only two. The resonance lines of the neutral atoms are clearly distinguished by their appearance as broad absorption lines against a continuous background.

\subsection{Ytterbium}

The first observations with this light source were made with ytterbium electrodes. Both the first and second spectra of this element have been extensively analyzed by W. F. Meggers, although only a small portion of the level structure has been reported[4].

Meggers compared these spectrograms with his $\mathrm{Yb}$ line-list and found that 23 lines of the first spectrum and 39 of the second spectrum were self-reversed. The highest level giving rise to self-reversed lines was at $19,710 \mathrm{~cm}^{-1}$ in $\mathrm{Yb} \mathrm{I}$ and at $35,832 \mathrm{~cm}^{-1}$ in $\mathrm{Yb}$ II.

Figure 2 shows the characteristic appearance of resonance lines in the first and second spectra of ytterbium. Both have wide gaps in the center of the emission line, but the wings of the $\mathrm{Yb}$ I line at $3464 \mathrm{~A}$ are almost completely absorbed. This shape appears to be characteristic of first spectrum resonance lines and can serve as a means of identifying them. The width of the self-absorbed center of this line was about $0.5 \mathrm{~A}$.

\subsection{Thulium}

The absorption spectrum of thulium has been observed by L. F. H. Bovey and W. R. S. Garton in an electric furnace operated at $2400{ }^{\circ} \mathrm{C}[5]$. They found 88 absorbed lines in the wavelength range 2600 to $6000 \mathrm{~A}$ which they interpreted as belonging to Tm I. However, a comparison with the partial analysis of Tm II reported by Meggers[6] reveals that 23 of these belong to this spectrum.

The spectrum obtained with the pulsed arc in the wavelength range 2300 to $7000 \mathrm{~A}$ contained 120 selfreversed lines of Tm I and 126 of Tm II. In Tm I only the ${ }^{2} \mathrm{~F}$ ground term and 16 higher levels have been reported by W. F. Meggers [6]. Every line which he designated as arising from a transition to the ${ }^{2} \mathrm{~F}_{7 / 2}$ ground state gives the appearance of a broad absorption line. However, there were seven more lines with the same character which could not be paired with a second line whose wave number difference equals the ${ }^{2} \mathrm{~F}$ interval. These have been classified by Meggers as originating from upper levels with $J=9 / 2$ and, as such, can only combine with the ${ }^{2} \mathrm{~F}_{7 / 2}$ ground state.

The line at $3916 \mathrm{~A}$ is due to a transition to the ${ }^{2} \mathrm{~F}_{5 / 2}$ level at $8771 \mathrm{~cm}^{-1}$ above the ground state. Although the absorbed center of the line is wide, the wings are strong and serve to distinguish these strongly self-reversed lines from the resonance lines.

The low ${ }^{3} \mathrm{~F}_{4,3,2}$ and ${ }^{1} \mathrm{~F}_{3}$ levels of $\mathrm{Tm}^{+}$(the ground state is ${ }^{3} \mathbf{F}_{4}$ ) were also found by Meggers [6]. Sixtyfive self reversed lines of Tm II are classified, among which are all but four lines of intensity greater than 50. This is a verification that the lines are correctly assigned. Meggers accepted only those upper levels which combined with three low levels. By using the self-reversed lines, the analysis can confidently be extended to include upper levels which combine with only two low levels. Also, upper levels making only one combination with a ${ }^{3} \mathrm{~F}$ level (because of the selection rule on $J$ ) can be confidently determined if the Zeeman pattern is correct and if the line is selfreversed.

\subsection{Uranium}

The spectrum of uranium in the range 2300 to $7000 \mathrm{~A}$ contained 100 self-reversed lines of $\mathrm{U}$ I and only 2 of U II. The absorbed central portions of the lines were much narrower than those of any of 

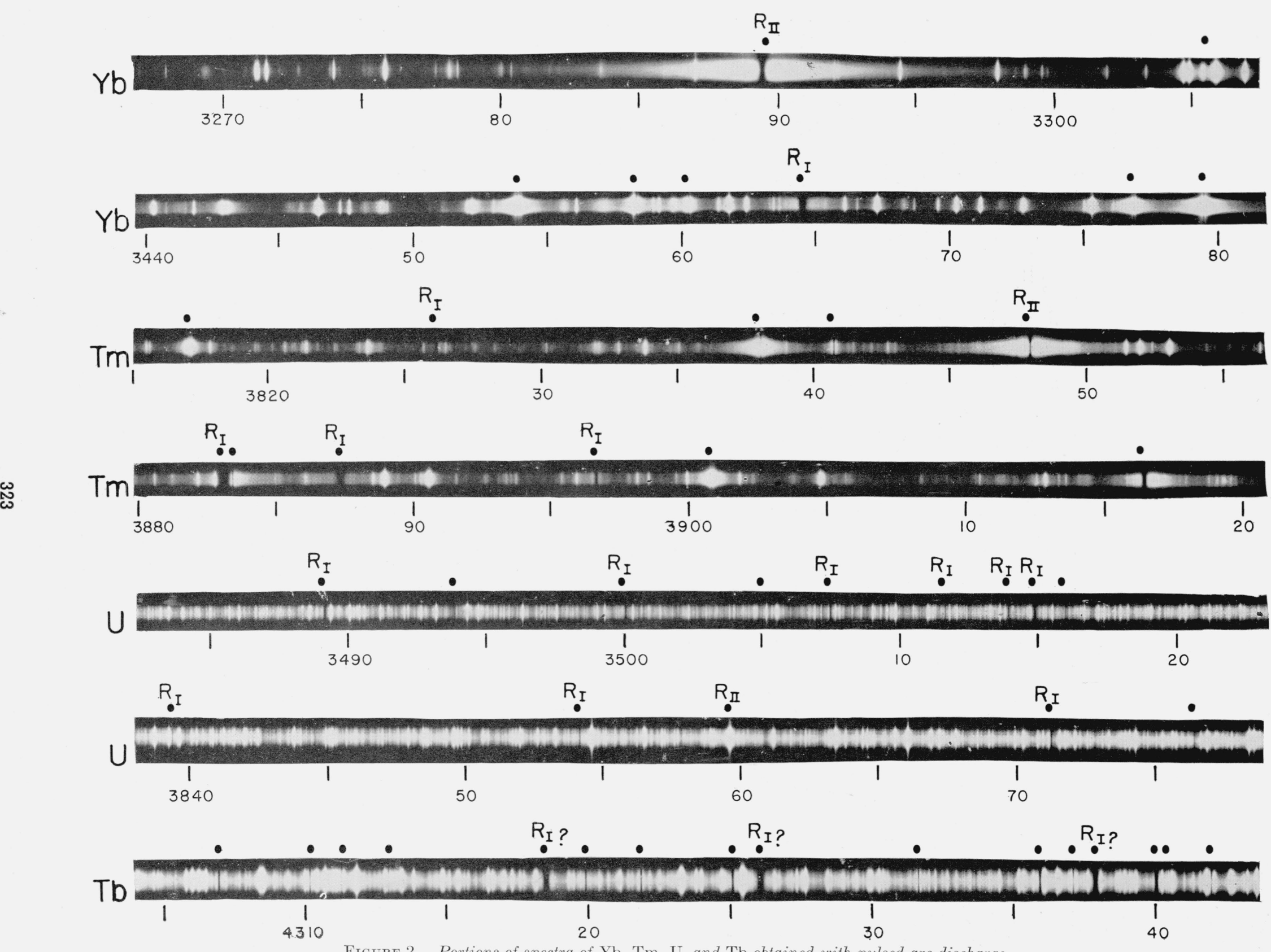
the other elements observed, although practically complete absorption of resonance lines was still present. None of these self-reversed lines classified by Kiess, Humphreys, and Laun [7] arise from transitions to levels higher than $3801 \mathrm{~cm}^{-1}$. However, many self-reversed lines are not classified, including resonance-type lines.

Recently a few U I lines were observed in absorption with a King-type furnace by N. P. Penkin and S. E. Frish [8].

In U II, two independent sets of energy levels are known. Neither of these has been established as the lower group. A listing of the levels and classified lines is given in a paper by Van Den Bosch [9], in which the two groups are referred to as $\mathrm{A}$ and $\mathrm{B}$.

The relative intensity tables of Meggers, Corliss, and Scribner [10] give the three leading strong lines of $\mathrm{U}_{\text {II }}$ as 3859,3854 , and 3670 A with intensities 360,180 , and 160 , respectively. The first and third of these are due to transitions in group B to levels at 289 and $915 \mathrm{~cm}^{-1}$, and are the only self-reversed lines in U II. The second line arises from a transition to the lowest level of group A. Because selfreversal depends on the product of the radiation intensity and the population of the absorbing energy level [3] for lines of approximately the same wavelength, the lowest level of group A must be higher than the $915 \mathrm{~cm}^{-1}$ level of group B. The ground state of $\mathrm{U}_{\text {II }}$ is therefore the ${ }^{4} \mathrm{I}_{9 / 2}$ level of the $5 f^{3} 6 s^{2}$ configuration in group B. No resonance lines of U II are self-reversed because they are too weak, the strongest having an intensity [10] of 120 .

The relatively weak self-reversal of uranium lines reflects the high boiling temperature of this element compared with those of the lanthanide series.

\subsection{Terbium}

Aside from a preliminary proposal by P. F. A. Klinkenberg [11] no analysis of the terbium spectrum has been published, but $J$ and $g$ values for a group of $\mathrm{Tb}$ I lines have been reported by S. Davis [12]. This spectrum was observed with the present light source to see whether any of the lines exhibiting very wide self-reversal had been analyzed by Davis.

About 400 lines in the region from 2300 to $7000 \mathrm{~A}$ showed self-reversal. Three of these strongly resemble resonance-type lines, with the emission lines almost completely absorbed. They appear in flgure 2 at 4318,4326 , and $4338 \mathrm{~A}$. Only the line at 4338 A has been assigned $J$ and $g$ values: $g_{1}=1.331$ $g_{2}=1.397, J_{1}=15 / 2, J_{2}=13 / 2$.

In pure LS-coupling the $g$-value for a ${ }^{6} \mathrm{H}_{15 / 2}$ term is 1.333 , and for a ${ }^{6} \mathrm{G}_{13 / 2}$ term is 1.385 . The most probable lowest configuration of $\mathrm{Tb} \mathrm{I}_{\mathrm{I}}$ is either $4 f^{9} 6 s^{2}$ or $4 f^{8} 5 d 6 s^{2}$. These would yield the ground states ${ }^{6} \mathrm{H}_{15 / 2}$ and ${ }^{8} \mathrm{H}_{17 / 2}$, respectively, according to Hund's rule. The strong self-reversal and Zeeman observations for the $4338 \mathrm{~A}$ line indicate that the ground state of $\mathrm{Tb}$ I is ${ }^{6} \mathrm{H}_{15 / 2}$. Furthermore, the upper state should belong to the $4 f^{8} 5 d 6 s^{2}$ configuration since Davis reported no indication of hyperfine structure for this line, nor for the other two strongly self-reversed lines mentioned above. This suggests that the $6 s$ shell is closed in both the upper and lower configurations.

This assignment is confirmed by Penselin and Schüpman [13] who observed the ${ }^{6} \mathrm{H}_{15 / 2}$ level by a magnetic resonance experiment, and obtained a $g$-value of 1.3225 . Since they also detected other levels, they could not conclude that this is the lowest state of $\mathrm{Tb}$.

The other two resonance-type lines are probably transitions from the excited ${ }^{6} \mathrm{H}_{15 / 2}$ and ${ }^{6} \mathrm{I}_{17 / 2}$ levels.

\section{Conclusion}

An important need in the problem of analysis of rare-earth spectra is now satisfied by the development of a simple light source giving self-reversed lines. While the analysis can probably be done without it, the initial break into the level structure is much more easily obtained with such information. Because of the unique appearance of absorbed resonance lines in the light source under discussion, these observations can verify whether or not the ground state of an atom has been found. Also, the amount of self-reversal of lines of the same intensity was shown to be a valuable clue for determining which absorbing energy level is lower.

This light source also produced numerous selfreversed lines in the second spectra of most of the observed elements.

\section{References}

[1] A. S. King, Astrophys. J. 60, 282 (1924).

[2] W. F. Meggers and O. Laporte, Phys. Rev. 28, 642 (1926).

[3] R. D. Cowan and G. H. Dieke, Rev. Mod. Phys. 20, 418 (1948).

[4] W. F. Meggers, J. Opt. Soc. Am. 50, 1136 (1960).

[5] L. F. H. Bovey and W. R. S. Garton, Proc. Phys. Soc. 6\%, 476 (1954)

[6] W. F. Meggers, Rev. Mod. Phys. 14, 96 (1942).

[7] C. C. Kiess, C. J. Humphreys, and D. D. Laun, J. Research NBS 3\%, 1 (1940) RP1729

[8] N. P. Penkin and S. E. Frish, Optika i Spek. 3, 473 (1957).

[9] J. C. Van Den Bosch, Physica 15, 503 (1949).

[10] W. F. Meggers, C. H. Corliss, and B. F. Scribner, Spectrochim Acta 1\%, 1168 (1961).

[11] P. F. A. Klinkenberg, Physica 13, 16 (1947).

[12] S. Davis, Astrophys. J. 132, 486 (1960).

[13] S. Penselin and K. Schüpman, unpublished reference in article by Cabezas, Lindgren, and Marrus, Phys. Rev. 122, 1797 (1961).

(Paper 66A4-166) 